\title{
Effect of L-thyroxine treatment on peripheral blood dendritic cell subpopulations in patients with Hashimoto's thyroiditis
}

\author{
Mariusz Stasiolek $^{1^{*}}$, Marek Dedecjus ${ }^{2 *}$, Zbigniew Adamczewski $^{1,3}$, \\ Przemyslaw Wiktor Sliwka ${ }^{1}$, Jan Brzezinski ${ }^{1}$, Andrzej Lewinski ${ }^{1,3}$
}

\author{
${ }^{1}$ Department of Endocrinology and Metabolic Diseases, Polish Mother's Memorial Hospital \\ - Research Institute, Lodz, Poland \\ ${ }^{2}$ Department of Oncological Endocrinology and Nuclear Medicine, Maria Sklodowska-Curie \\ Memorial Cancer Centre and Institute of Oncology, Warsaw, Poland \\ ${ }^{3}$ Medical University of Lodz, Poland
}

\begin{abstract}
Recent reports suggested dendritic cells (DCs) to be important players in the pathogenesis of autoimmune thyroid processes in humans. However, there are virtually no data addressing the influence of thyroid autoaggression-associated disturbances of thyrometabolic conditions on DCs biology. The aim of the study was to evaluate the influence of L-thyroxine supplementation on conventional and plasmacytoid peripheral blood DCs subtypes in patients with hypothyroidism due to Hashimoto's thyroiditis (HT). Eighteen patients with newly diagnosed hypothyroidism due to HT were included into the study. All patients received L-thyroxine treatment with doses adjusted to reach euthyroidism. Peripheral blood DC subtypes structure and immunoregulatory phenotype were analyzed by flow cytometry in the same patient prospectively at two time points: (i) before and (ii) 3 months after beginning of L-thyroxine treatment (hypothyroidism vs. euthyroidism, respectively). Percentage of plasmacytoid DCs in peripheral blood mononuclear cells fraction was significantly decreased in the course of L-thyroxine treatment $(0.27 \pm 0.19$ vs. $0.11 \pm 0.08 ; p<0.05)$, whereas we did not observe any changes in the number of conventional DCs. However, the phenotypic analysis showed a significant increase of conventional DCs expressing CD86 and CD91 (64.25 $\pm 21.6 \%$ vs. $86.3 \pm 11 \%$; $p<0.05$ and $30.75 \pm$ $\pm 11.66 \%$ vs. $44.5 \pm 13.3 \%$; $\mathrm{p}<0.05$; respectively) in euthyroid patients. Standard L-thyroxine supplementation in HT patients exerted significant immunoregulatory effects, associated with quantitative and phenotypic changes of peripheral blood DC subpopulations. (Folia Histochemica et Cytobiologica 2014, Vol. 52, No. 2, 138-143)
\end{abstract}

Keywords: Hashimoto's thyroiditis; L-thyroxine; hypothyroidism; autoimmunity; dendritic cells; CD86; CD91

\section{Introduction}

Dendritic cells (DCs) are considered to be the most efficient antigen presenting cells (APCs) and crucial regulators of primary and adaptive immune response. Depending on their subtype, maturation

\footnotetext{
Correspondence address: A. Lewinski,

Department of Endocrinology and Metabolic Diseases,

Polish Mother's Memorial Hospital — Research Institute

281/289 Rzgowska St., 93-338 Lodz, Poland;

tel.: +48 4227111 41, fax: +48 4227111 40;

e-mail: alewin@csk.umed.lodz.pl

*Both authors contributed equally to this work
}

state and microenvironmental factors (including vast variety of cytokines, hormones and the interaction with other immune and non-immune cells) DCs are able to initiate, direct and control different kinds of inflammatory reaction, as well as to regulate immune tolerance mechanisms, both on central and peripheral levels $[1,2]$. In humans there are two main subtypes of peripheral blood DCs - myeloid (or conventional) DCs (cDCs) and plasmacytoid DCs (pDCs), which differ substantially in phenotype and functional characteristics [3]. Due to their powerful immunoregulatory properties, both populations of peripheral blood DCs have been implicated in pathogenesis of numerous human diseases of inflammatory origin, including 
lupus erythematosus [4] and multiple sclerosis [5] and in mechanisms of immune escape in different malignancies [6]. Similarly, DCs are considered to play an important role already in the earliest phases of pathological events, leading to the autoimmune thyroid disease (AITD) in humans [7] and spontaneous autoimmune thyroid disease in BB rats [8], as well as in the immune response mounted against thyroid cancer [9, 10, reviewed extensively in 11]. Interestingly, results of animal studies suggested also that different populations of DCs exerted diverse and even contradictory effects on the development and clinical course of experimental autoimmune thyroiditis (EAT) $[12,13]$. In accordance with EAT data, the most recent findings suggest one of the specific regulatory DC subsets, pDCs to be a crucial cell population in thyroid autoaggression in humans [14]. In the light of the well-known interplay between hormonal and immune systems, it seems to be of great importance to characterize the influence of thyrometabolic conditions on the inflammatory processes of AITD. In our previous work, performed on patients who were thyroidectomized because of differentiated thyroid cancer, we showed - for the first time - that thyroid hormones are important physiological regulators of naturally occurring human peripheral blood $\mathrm{pDCs}$ and cDCs, both in vivo and in vitro [15]. Additionally, such DC-associated regulatory effects were not observed in another group of thyroidectomized patients receiving systemically exogenous thyrotropin (TSH), which seems to further support the direct, independent of the TSH concentration fluctuations, influence of thyroid hormones on human peripheral blood DCs in vivo [16]. These findings, together with the analysis performed by Leskela and colleagues [14], arise a question about possible DC-dependent immunomodulatory effects of L-thyroxine (L-T4) supplementation in patients with hypothyroidism due to Hashimoto's thyroiditis (HT). In our study, we assessed prospectively proportions and phenotype of peripheral blood conventional and plasmacytoid DCs at two consecutive time points in the same HT patient: (i) before beginning of L-T4 supplementation, i.e. in a state of hypothyroidism, and (ii) after 3 months of sufficient L-T4 administration, i.e. in euthyroid state. Our analysis showed that L-T4 supplementation in HT not only normalized thyrometabolic parameters, but also exerted significant quantitative and qualitative effects on both main peripheral blood DCs subtypes.

\section{Material and methods}

Patients. Venous blood samples, collected from patients with HT $(n=18)$ were submitted to analysis. The patients
Table 1. Clinical characteristics of study participants

\begin{tabular}{|l|c|c|}
\hline & $\begin{array}{c}\text { Before L-T4 } \\
\text { treatment } \\
(\mathbf{n}=\mathbf{1 8})\end{array}$ & $\begin{array}{c}\text { After 3 months } \\
\text { of L-T4 treatment } \\
(\mathbf{n}=\mathbf{1 8})\end{array}$ \\
\hline Age $(\mathrm{years})$ & $46.1 \pm 13.17$ & $46.1 \pm 13.17$ \\
\hline $\mathrm{BMI}\left[\mathrm{kg} / \mathrm{m}^{2}\right]$ & $25.6 \pm 4.6$ & $24.9 \pm 4.4^{\mathrm{a}}$ \\
\hline $\mathrm{FT}_{4}[\mathrm{pmol} / \mathrm{ml}]$ & $6.5 \pm 2.3$ & $19.7 \pm 3.6^{\mathrm{a}}$ \\
\hline $\mathrm{FT}_{3}[\mathrm{pg} / \mathrm{ml}]$ & $1.1 \pm 0.26$ & $4.21 \pm 1.05^{\mathrm{a}}$ \\
\hline $\mathrm{TSH}(\mathrm{mIU} / \mathrm{l})$ & $47.26 \pm 20.3$ & $1.4 \pm 1.2^{\mathrm{a}}$ \\
\hline $\mathrm{TgAb}[\mathrm{IU} / \mathrm{ml}]$ & $910 \pm 650$ & $814 \pm 760$ \\
\hline $\mathrm{TPOAb}[\mathrm{IU} / \mathrm{ml}]$ & $440 \pm 270$ & $320 \pm 350^{\mathrm{a}}$ \\
\hline
\end{tabular}

${ }^{\mathrm{a}} \mathrm{p}<0.05$

were recruited from the Department of Endocrinology and Metabolic Diseases, Polish Mother's Memorial Hospital - Research Institute, Lodz, Poland. Only the patients with newly diagnosed hypothyroidism due to HT, with no history of L-T4 administration, were qualified for the study. Any concomitant immunological or metabolic disorders (i.e. diabetes mellitus) were regarded as exclusion criteria (for further patients characteristics see Table 1). All of the patients signed the informed consent, and the Ethics Committee of the Medical University of Lodz had approved the study protocol. Blood samples were collected between 08.00 and $09.00 \mathrm{AM}$ after an overnight fast. Venous blood was obtained by clean venipuncture (needle gauge 19), avoiding slow flowing draws and/or traumatic venipunctures. The samples were collected from the same patient at two consecutive time points: (i) before commencement of L-T4 supplementation - hypothyroidism (mean value of $\mathrm{TSH}-47.26 \pm 20.3 \mathrm{mIU} / \mathrm{l}$ ), and (ii) after 3 months of L-T4 administration - euthyroidism (mean value of TSH $-1.4 \pm$ $\pm 1.2 \mathrm{mIU} / \mathrm{l})$.

Free triiodothyronine (FT3), free thyroxine (FT4) and TSH concentrations were measured by the immunoradiometric (IRMA) method with appropriate kits (BRAHMS, Berlin, Germany; normal values: TSH 0.3-4.0 mIU/1; FT 3.4-7.65 pmol/l; $\left.\mathrm{FT}_{4} 10-25 \mathrm{pmol} / \mathrm{l}\right)$. The concentrations of $\mathrm{TgAb}$ and TPOAb were measured by the electrochemiluminescence (ECLIA) method with appropriate kits (Roche Diagnostics Mannheim, Germany, normal values: $\mathrm{TgAb}$ $<115 \mathrm{IU} / \mathrm{ml} ; \mathrm{TPOAb}<35 \mathrm{IU} / \mathrm{ml}$ ) and equipment (Modular Analytics E170 - Roche Diagnostic).

Fluorescence-activated cell sorting (FACS) analysis. Peripheral blood mononuclear cells (PBMCs) were isolated from blood samples (obtained as described above) by centrifugation on a discontinuous density gradient (Histopaque 1077, SigmaAldrich, St. Louis, MO, USA). PBMCs were assessed by flow cytometry, using a FACSCalibur ${ }^{\circledast}$ cytometer and CELLQuest ${ }^{\circledR}$ software (BD Biosciences, San Jose, CA, 

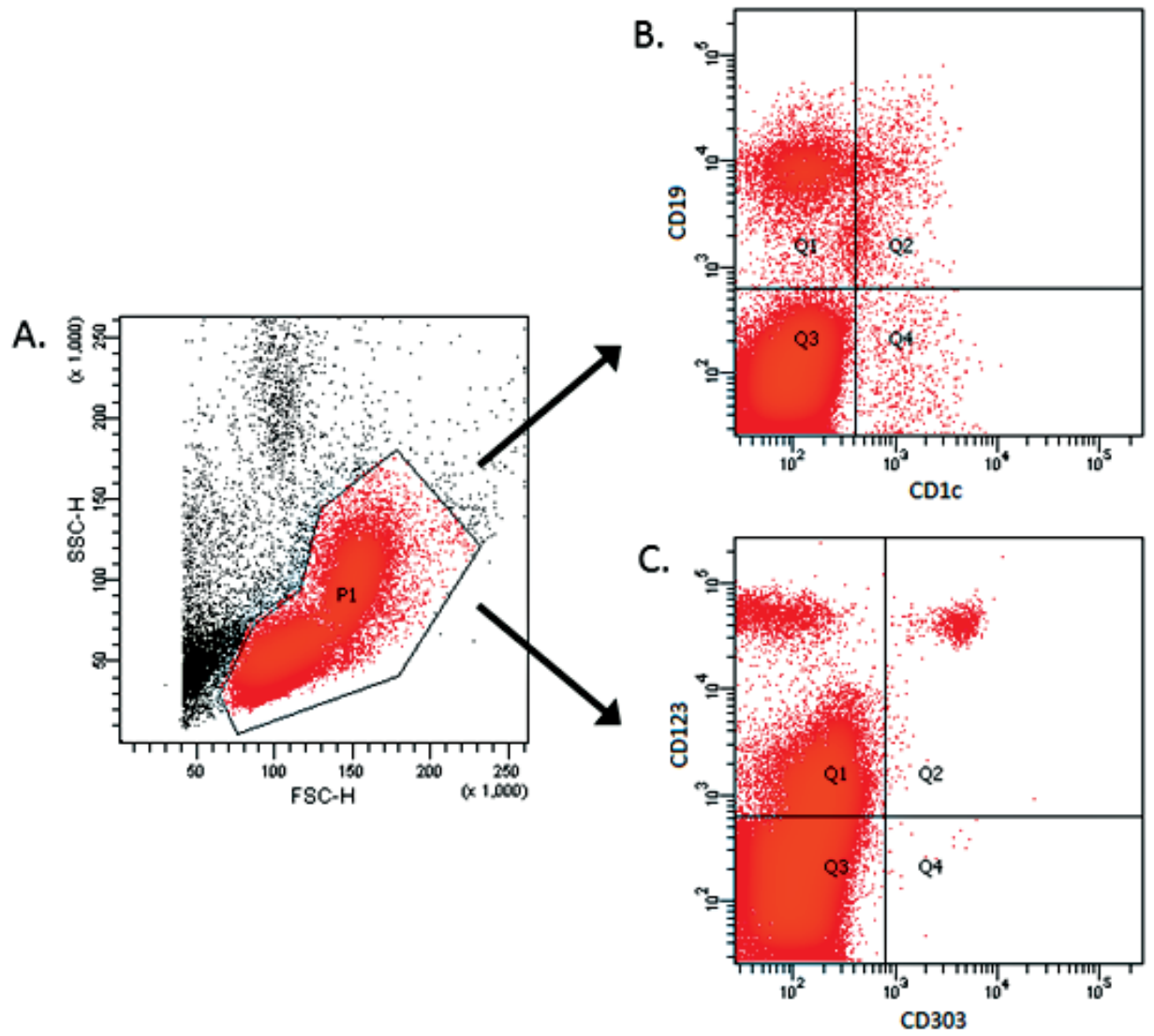

Figure 1. Representative dot plots of FACS analysis. A. Distribution of cellular populations in forward and side scatter (FSC-SSC) picture, PBMCs were gated for further DC analysis (gate P1). B. BDCA1/CD1c+CD19- cDCs (quadrant Q4).C. BDCA2/CD303+CD123+ pDCs (quadrant Q2)

USA). Peripheral blood DCs were recognized on the basis of surface expression of appropriate blood dendritic cell antigens (BDCAs) using fluorescein isothiocyanate (FITC) conjugated monoclonal antibodies (mAb) specific for BDCA1/CD1c and BDCA2/CD303. The conventional myeloid subset of DCs was defined as a population positive for BDCA1/CD1c (anti-CD1c-Ab, AD5-8E7, mouse IgG2a) and negative for CD19 (in order to exclude CD1c positive CD19+ B cells; phycoerythrin-cyanie dye (PE-Cy5) conjugated anti-CD19-Ab, HIB19, mouse IgG1). Plasmacytoid DCs were recognized as BDCA2/CD303 positive cells (anti-CD303-Ab, AC144, mouse IgG1). All DC populations were counterstained with phycoerythrin (PE) conjugated mAb: anti-HLA-DR (G46-6 (L243), mouse IgG2a), anti-CD11c (B-ly6, mouse IgG1), anti-CD40 (5C3, mouse IgG1), anti-CD80 (L307.4, mouse IgG1), anti-CD83 (HB15e, mouse IgG1), anti-CD86 (2331(FUN-1), mouse IgG1), anti-CD91 (A2MR- $\alpha 2$, mouse IgG1), anti-CD123 (9F5, mouse IgG1). Monoclonal Ab specific for BDCA antigens, were purchased from Miltenyi Biotec (Bergisch Gladbach, Germany). All the other mAb and appropriate isotype controls were purchased from BD Biosciences Pharmingen (San Jose, CA, USA). In each sample $5 \times 10^{5}$ cells were stained $(\mathrm{mAb}$ concentration in each case as provided in " 1 test" by manufacturer) and $2 \times 10^{5}$ cells were collected in order to gain at least $1 \times 10^{5}$ cells in PBMC gate. The results of FACS analysis were presented as percentage of positive cells in the whole PBMCs fraction. Figure 1 shows representative FACS analysis of plasmacytoid and conventional DCs.

Statistical analysis. Statistical analyses were performed, using the STATGRAPHICS PLUS v. 5.0 software. Student's $t$-test for paired samples was used to determine the significance of differences in all the measured parameters with normal distribution, observed between patients before and during L-T4 therapy. For the data that were not normally distributed, nonparametric Wilcoxon's rank test was used to determine the statistical significance of differences. P-value less than 0.05 was considered statistically significant.

\section{Results}

The quantitative flow cytometric analysis showed that the percentage of pDCs in the whole PBMCs fraction significantly decreased in the course of L-T4 treatment $(0.27 \pm 0.19$ vs. $0.11 \pm 0.08 ; \mathrm{p}<0.05)$. 


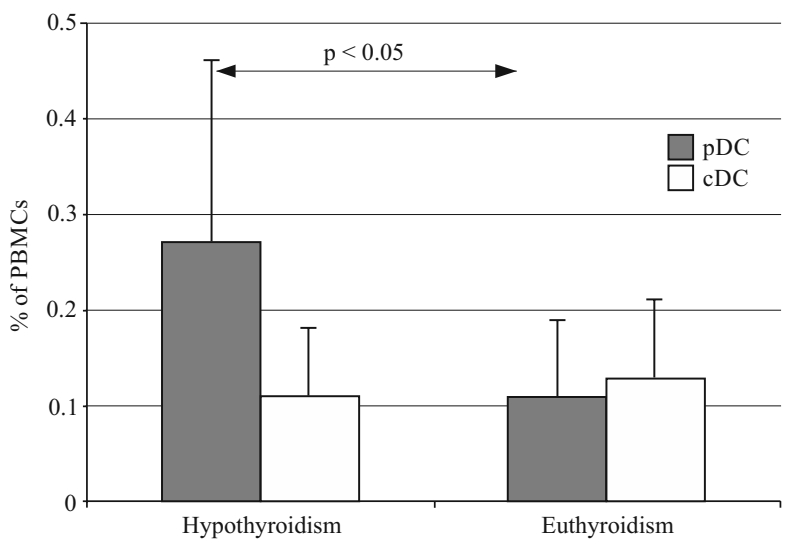

Figure 2. The structure of peripheral blood DC subtypes (pDC and cDCs) was analyzed in the same HT patient $(n=18)$ before and 3 months after commencement of L-T4 treatment (hypothyroidism and euthyroidism, respectively). Bars represent mean values \pm SD

Interestingly, we did not observe any quantitative effects of L-T4 treatment on cDCs population (Figure 2). However, the assessment of DC surface expression profile in the same patient before and during L-T4 supplementation demonstrated a significant increase of cDCs expressing CD86 - one of the most important costimulatory molecules $(64.25 \pm 21.6 \% \mathrm{vs} .86 .3 \pm$ $\pm 11 \% ; \mathrm{p}<0.05)$. Also, the surface expression of CD91 was increased on cDCs in euthyroid patients, as compared to the hypothyroid state $(30.75 \pm 11.66 \% \mathrm{vs}$. $44.5 \pm 13.3 \%$; $\mathrm{p}<0.05$ ) (Figure 3 ). To the contrary, the expression of both CD86 and CD91 on pDCs remained unchanged during our study. We did not observe any effects of L-T4 administration on the surface expression of HLA-DR and other investigated immunoregulatory markers (CD40, CD80, CD83, CD200), either in conventional or plasmacytoid DCs population (data not shown).

\section{Discussion}

The main finding of our prospective study is the modulatory effect of L-T4 supplementation on both plasmacytoid and conventional peripheral blood DCs subpopulations in HT patients. The involvement of DCs in the pathogenesis of various forms of autoimmune thyroid disease seems to be increasingly well documented [17]. However, there are still very little data based on human studies available. The analysis of transcriptomic profiles of thyroid tissue in different stages of Graves' disease (GD) revealed progressive accumulation of changes in the expression of numerous immune factors, among them - associated with pDCs [18]. On the cellular level, it has been shown that

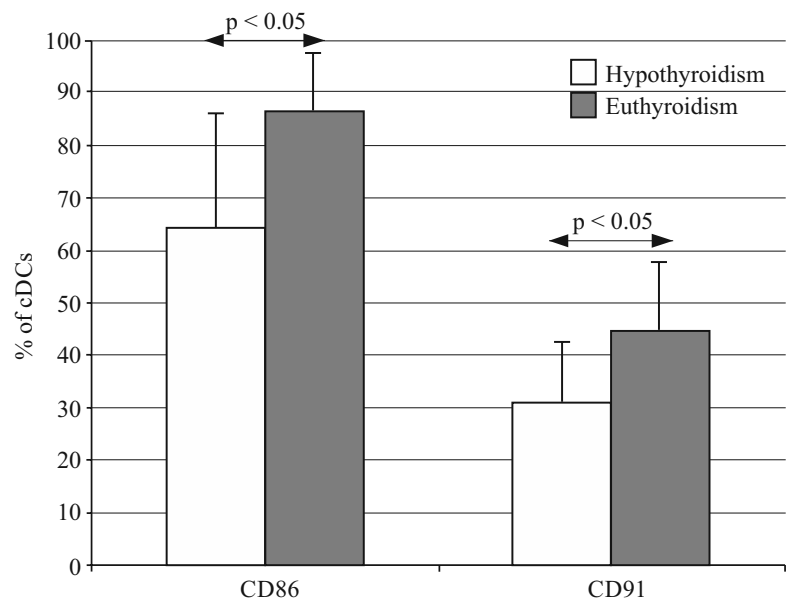

Figure 3. The surface expression of immunomodulatory molecules CD86 and CD91 was analyzed in the same HT patient $(n=18)$ before and 3 months after commencement of L-T4 treatment (i.e. hypothyroidism and euthyroidism, respectively). Bars represent mean values $\pm \mathrm{SD}$

the decrease of regulatory $\mathrm{T}$ cells (Tregs) observed in peripheral blood of untreated GD patients was associated with elevated $\mathrm{pDC} / \mathrm{cDC}$ proportion [19]. Moreover, in co-culture experiments the authors demonstrated that $\mathrm{pDCs}$ (but not cDCs) derived from untreated GD patients efficiently inhibited immunosuppressive activity of Tregs [19]. Most recently, Leskela and colleagues [14] have shown decreased percentage of pDCs in PBMCs obtained both from patients with HT and GD, as well as disease- and disease-stage specific changes in immunoregulatory phenotype of pDCs. These peripheral blood findings were accompanied by increased accumulation of pDCs observed in thyroid tissue specimens from HT and GD patients [14]. Another research group has reported recently significant increase of thyroid infiltrating pDC in untreated GD patients [7] and patients with HT. However, in HT these parameters did not depend on disease clinical stage [20].

Although the results published so far clearly point at pDCs as the population with crucial engagement in autoimmune mechanisms underlying various forms of human AITD associated with different hormonal parameters, none of the above mentioned studies encompassed a prospective analysis of the influence of thyrometabolic state on DCs in AITD patients. In our study, we analyzed ex vivo peripheral blood pDC and cDCs populations in the same HT patients before (in hypothyroid state) and 3 months after beginning of L-T4 supplementation (in euthyroid state). In concordance with the results presented by Leskela and colleagues [14], we observed low percentage 
of pDCs in our group of HT patients, however this parameter was further decreased in the course of L-T4 treatment. The percentage of cDCs remained unchanged after normalization of TSH and thyroid hormones levels. Still, in that population we observed an increased expression of CD86, whereas the expression of CD80 remained very low and unaffected by L-T4 supplementation. The molecules CD80 and CD86 belong to the most important members of B7 family of costimulatory molecules and play a crucial role in the time- and stimulation-dependent regulatory loop of immune response. The CD86 molecule, present already on resting DCs, delivers the main costimulatory signal in the earliest stage of immune reaction. To the contrary, CD80 expressed first after previous proinflammatory stimulation is considered to exert its main action in later phases of immune response [21]. Thus, the increased expression of CD86 without change in CD80 status may rather represent a different basal functional characteristics of cDCs than a sign of undergoing proinflammatory stimulation. Also, the increased expression of CD91, molecule known to act as heat shock protein receptor involved in cross presentation and $\mathrm{T}$ cell priming [22], may influence the basic ability and readiness of peripheral blood $\mathrm{cDCs}$ to react upon danger signal encounter. Taking together, these observations may suggest that thyrometabolic parameters affect in an unspecific manner immune parameters in the periphery, including modulation of various DC populations. However, in particular clinical situations, such as HT, this influence may differ, especially in the case of immune cell populations specifically engaged in the immunopathological events. This assumption could possibly explain why the effects exerted in vivo by L-T4 on peripheral blood DCs in patients thyroidectomized because of differentiated thyroid cancer [15], differed from the results obtained in HT, most remarkably in the case of pDCs, cell population postulated as one of the most important elements of autoimmune process in this disease.

The observed modulatory effect of L-T4 on CD86 expression on human peripheral blood cDCs seems to stay in line with the animal studies, which demonstrated that the effects of thyroid hormones exerted on murine bone marrow-derived DCs in culture included modulation of CD86 expression and were associated with thyroid hormone receptor dependent activation of NF- $\kappa \mathrm{B}$ and Akt signaling [23, 24]. Moreover, these immunoregulatory effects of thyroid hormones could be inhibited by glucocorticosteroids in mechanism based on the down-regulation of the same transcriptional pathways [25].
DCs have been suggested in some experimental studies to correlate with the appearance of anti-thyroid antibodies [26]. However, we think that the decrease of anti-Tg Ab serum level observed in the course of L-T4 supplementation in our patients' group may not be directly attributed to changes in peripheral blood DCs. In the light of the unclear etiopathological role of anti-Tg Abs this problem needs a large population-based study to be resolved definitely.

We think that our results not only stay very well in concordance with the latest report regarding the role of DCs in AITD in humans [14] but also supplement significantly its findings. Taking into consideration the very high frequency of AITD [27] and common administration of drugs modifying thyroid hormones activity, we believe that the influence of thyrometabolic conditions on immune system and - in particular - on DC function represents a very important issue of modern endocrinology and immunology and definitely deserves more attention both in clinical and laboratory medicine.

\section{Acknowledgments}

The study was financially supported by statutory funds (2013/VII/22) from the Polish Mother's Memorial Hospital - Research Institute, Lodz, Poland.

\section{References}

1. Lipscomb MF, Masten BJ. Dendritic cells: immune regulators in health and disease. Physiol Rev. 2002;82:97-130.

2. Stasiołek M. The role of selected immunoregulatory cell populations in autoimmune demyelination. Neuro Endocrinol Lett. 2011;32:25-33.

3. Liu K, Nussenzweig MC. Origin and development of dendritic cells. Immunol Rev. 2010;234:45-54.

4. Jin O, Kavikondala S, Sun L et al. Systemic lupus erythematosus patients have increased number of circulating plasmacytoid dendritic cells, but decreased myeloid dendritic cells with deficient CD83 expression. Lupus. 2008;17:654-662.

5. Stasiolek M, Bayas A, Kruse N et al. Impaired maturation and altered regulatory function of plasmacytoid dendritic cells in multiple sclerosis. Brain. 2006;129:1293-1305.

6. Faget J, Bendriss-Vermare N, Gobert M et al. ICOS-ligand expression on plasmacytoid dendritic cells supports breast cancer progression by promoting the accumulation of immunosuppressive CD4+ T cells. Cancer Res. 2012;72:6130-6141.

7. Hammerstad SS, Jahnsen FL, Tauriainen S et al. Inflammation and increased myxovirus resistance protein a expression in thyroid tissue in the early stages of Hashimoto's thyroiditis. Thyroid. 2013;23:334-341.

8. Voorby HA, Kabel PJ, de Haan M et al. Dendritic cells and class II MHC expression on thyrocytes during the autoimmune thyroid disease of the BB rat. Clin Immunol Immunopathol. 1990;55:9-22.

9. Yu H, Huang X, Liu X et al. Regulatory T cells and plasmacytoid dendritic cells contribute to the immune escape of papillary thyroid cancer coexisting with multinodular non-toxic goiter. Endocrine. 2013;44:172-181. 
10. Hilly O, Koren R, Raz R et al. The role of s100-positive dendritic cells in the prognosis of papillary thyroid carcinoma. Am J Clin Pathol. 2013;139:87-92.

11. Lewiński A, Śliwka PW, Stasiołek M. Dendritic cells in autoimmune disorders and thyroid cancer. Folia Histochem Cytobiol. 2014;52:18-28.

12. Vasu C, Dogan RN, Holterman MJ, Prabhakar BS. Selective induction of dendritic cells using granulocyte macrophage-colony stimulating factor, but not fms-like tyrosine kinase receptor 3-ligand, activates thyroglobulin-specific CD4+/CD25+ $\mathrm{T}$ cells and suppresses experimental autoimmune thyroiditis. J Immunol. 2003;170:5511-5522.

13. Gangi E, Vasu C, Cheatem D, Prabhakar BS. IL-10-producing $\mathrm{CD} 4+\mathrm{CD} 25+$ regulatory $\mathrm{T}$ cells play a critical role in granulocyte-macrophage colony-stimulating factor-induced suppression of experimental autoimmune thyroiditis. $J$ Immunol. 2005;174:7006-7013.

14. Leskela S, Rodríguez-Muñoz A, de la Fuente $\mathrm{H}$ et al. Plasmacytoid dendritic cells in patients with autoimmune thyroid disease. J Clin Endocrinol Metab. 2013;98:2822-2833.

15. Dedecjus M, Stasiolek M, Brzezinski J, Selmaj K, Lewinski A. Thyroid hormones influence human dendritic cells' phenotype, function, and subsets distribution. Thyroid. 2011;21:533-540.

16. Stasiolek M, Adamczewski Z, Puła B et al. Distribution of subpopulations of dendritic cells in peripheral blood of patients treated with exogenous thyrotropin. Thyroid Res. 2012;5:18.

17. Ganesh BB, Cheatem DM, Sheng JR, Vasu C, Prabhakar BS. GM-CSF-induced CD11c+CD8a - dendritic cells facilitate Foxp3+ and IL-10+ regulatory T cell expansion resulting in suppression of autoimmune thyroiditis. Int Immunol. 2009;21:269-282.

18. Ruiz-Riol M, Barnils Mdel P, Colobran Oriol R et al. Analysis of the cumulative changes in Graves' disease thyroid glands points to IFN signature, plasmacytoid DCs and alternatively activated macrophages as chronicity determining factors. J Autoimmun. 2011;36:189-200.

19. Mao C, Wang S, Xiao Y et al. Impairment of regulatory capacity of $\mathrm{CD} 4+\mathrm{CD} 25+$ regulatory $\mathrm{T}$ cells mediated by dendritic cell polarization and hyperthyroidism in Graves' disease. J Immunol. 2011;186:4734-4743.

20. Hammerstad SS, Jahnsen F, Tauriainen S et al. Immunological changes and increased expression of myxovirus resistance protein a in thyroid tissue of patients with recent onset and untreated Graves' Disease. Thyroid. 2013;24:537-544 .

21. Chambers CA. The expanding world of co-stimulation: the two-signal model revisited. Trends Immunol. 2001;22: 217-223.

22. Pawaria S, Binder RJ. CD91-dependent programming of T-helper cell responses following heat shock protein immunization. Nat Commun. 2011;2:521.

23. Mascanfroni I, Montesinos Mdel M, Susperreguy S et al. Control of dendritic cell maturation and function by triiodothyronine. FASEB J. 2008;22:1032-1042.

24. Mascanfroni ID, Montesinos Mdel M, Alamino VA et al. Nuclear factor (NF)-kappaB-dependent thyroid hormone receptor beta1 expression controls dendritic cell function via Akt signaling. J Biol Chem. 2010;285:9569-9582.

25. Montesinos MM, Alamino VA, Mascanfroni ID et al. Dexamethasone counteracts the immunostimulatory effects of triiodothyronine (T3) on dendritic cells. Steroids. 2012;77: $67-76$.

26. Li HS, Verginis P, Carayanniotis G. Maturation of dendritic cells by necrotic thyrocytes facilitates induction of experimental autoimmune thyroiditis. Clin Exp Immunol. 2006;144:467-474.

27. McLeod DS, Cooper DS. The incidence and prevalence of thyroid autoimmunity. Endocrine. 2012;42:252-265.

Submitted: 8 January, 2014 Accepted after reviews: 15 May, 2014 